\title{
Overexpression of the Trichoderma brevicompactum tri5 Gene: Effect on the Expression of the Trichodermin Biosynthetic Genes and on Tomato Seedlings
}

\author{
Anamariela Tijerino ${ }^{1}$, Rosa Hermosa ${ }^{1}$, Rosa E. Cardoza ${ }^{2}$, Javier Moraga ${ }^{3}$, Monica G. Malmierca ${ }^{2}$, \\ Josefina Aleu ${ }^{3}$, Isidro G. Collado ${ }^{3}$, Enrique Monte ${ }^{1}$ and Santiago Gutierrez ${ }^{2}$ * \\ 1 Spanish-Portuguese Center of Agricultural Research (CIALE), Department of Microbiology and \\ Genetics, University of Salamanca, Campus of Villamayor, Río Duero 12, 37185 Salamanca, Spain; \\ E-Mails: atijerino@usal.es (A.T.); rhp@usal.es (R.H.); emv@usal.es (E.M.) \\ 2 University of León, Campus of Ponferrada, Superior and Technical Universitary School of \\ Agricultural Engineers, Area of Microbiology, Avda. Astorga s/n, 24400 Ponferrada, Spain; \\ E-Mails: recars@unileon.es (R.E.C.); mgomh@unileon.es (M.G.M.) \\ 3 University of Cádiz, Puerto Real, Science Faculty, Department of Organic Chemistry, \\ 11510 Cádiz, Spain; E-Mails: javier.moraga@uca.es (J.M.); josefina.aleu@uca.es (J.A.); \\ isidro.gonzalez@uca.es (I.G.C.) \\ * Author to whom correspondence should be addressed; E-Mail: s.gutierrez@ unileon.es; \\ Tel.: +34-987442060; Fax: +34-987442070.
}

Received: 7 September 2011; in revised form: 15 September 2011 / Accepted: 21 September 2011 / Published: 23 September 2011

\begin{abstract}
Trichoderma brevicompactum IBT 40841 produces trichodermin, a trichothecene-type toxin that shares most of the steps of its biosynthesis with harzianum A, another trichothecene produced by several Trichoderma species. The first specific step in the trichothecene biosynthesis is carried out by a terpene cylcase, trichodiene synthase, that catalyzes the conversion of farnesyl pyrophosphate to trichodiene and that is encoded by the tri5 gene. Overexpression of tri5 resulted in increased levels of trichodermin production, but also in an increase in tyrosol and hydroxytyrosol production, two antioxidant compounds that may play a regulatory role in trichothecene biosynthesis, and also in a higher expression of three trichothecene genes, tri4, tri6 and tri10, and of the erg1 gene, which participates in the synthesis of triterpenes. The effect of tri5 overexpression on tomato seedling disease response was also studied.
\end{abstract}


Keywords: trichothecene; terpene; tyrosol; tri genes; ergosterol; gene expression

\section{Introduction}

Trichoderma species are well known as biocontrol agents of plant diseases in a broad variety of crops [1], and their ability to produce a huge diversity of antimicrobial compounds and cell wall degrading enzymes has been extensively studied and reviewed [2-5]. Among the secondary metabolites produced by Trichoderma, the terpenes include hundreds of different compounds with a huge diversity of physiological activities. Terpenes arise from the repetitive fusion of branched five-carbon units based on an isopentane skeleton, and most of the chemical intermediates in their biosynthetic pathway are known. In fungi, the first enzyme involved in the terpene pathway is 3-hydroxy-3-methylglutaryl-coenzyme A reductase (HMG-CoA reductase), which metabolizes HMG-CoA to mevalonic acid (MVA). Subsequently, the isomerization of mevalonate produces isopentenyl diphosphate (IPP), which condenses with dimethylallyl diphosphate to produce geranyl diphosphate (GPP), the key precursor of monoterpene biosynthesis. This molecule is condensed again with IPP, affording farnesyl diphosphate (FPP), a common intermediate in the production of: (i) geranylgeranyl diphosphate (GGPP) and its diterpene derivatives; (ii) trichodiene and the subsequent sesquiterpene compounds, including trichothecene fungal toxins; and (iii) squalene, as a precursor of the triterpene pathway to produce prenylated proteins, sterols (ergosterol), ubiquinones, dolichols and other secondary metabolites [6,7] as well as plant carotenoids, gibberellins, tocopherols and chlorophylls [8].

The biosynthesis of trichothecenes has mainly been documented in Fusarium species, and their biosynthesis has been extensively reviewed [9,10]. Recently, most of the steps involved in the trichothecene biosynthesis in Trichoderma species have been described and the genes characterized [11]. Trichoderma brevicompactum has been classified within the clade Brevicompactum [12], which includes species with a common pathway from FPP to trichodermol. From trichodermol the pathway diverges to produce harzianum $\mathrm{A}$ in $T$. arundinaceum, $T$. turrialbense and $T$. protrudens or trichodermin, in T. brevicompactum. The tri5 gene, involved in the biosynthesis of trichodiene from FPP, has recently been cloned and characterized [11,13]. Additionally, another 7 genes involved in trichodermin biosynthesis have been identified in the T. brevicompactum genome. These genes are grouped in a core cluster [11]. Interestingly, the tri5 gene is located outside this cluster, being an exception of the trichothecene biosynthetic clusters described until now in Fusarium [14] and Myrothecium [15] where tri5 gene is located inside the clusters.

Transformants of $T$. brevicompactum IBT 40841, with a higher level of tri5 gene expression, had higher levels of trichodermin production and greater antibiotic activity against several yeast strains. A significant increase in the level of production of the antioxidants tyrosol and hydroxytyrosol was also detected in the tri5-overexpressing transformants [13]. Tyrosol is a phenolic antioxidant metabolite that is present in olive oil and wine [16], yeast [17], and T. viride [18] and T. brevicompactum cultures [13]. It is synthesized from a transamination reaction of L-tyrosine and 2-oxoglutarate, giving rise to L-glutamic acid and p-hydroxyphenyl-pyruvate. The later product is then decarboxylated, and the resulting aldehyde is reduced to tyrosol [19-21]. It has been shown that the tyrosol acts as a 
quorum-sensing molecule, which accelerates the morphological transition from yeasts to hyphae and facilitates biofilm production [17]. Hydroxytyrosol is a derivative of tyrosol with antioxidant properties due to its ortho-dihydroxyphenolic group [19]. The relationship between the tyrosol/hydroxytyrosol and trichodermin is not clear and needs additional studies.

In the present article, we report the effect of tri5 gene overexpression on the expression of other tri genes in minimal medium and in media containing the antioxidant tyrosol. The effect of tri5 overexpression on expression of the ergl gene, which is involved in the synthesis of triterpene compounds [22], was also determined. Finally, the effect of tri5 overexpression on tomato seedling disease response was examined.

\section{Materials and Methods}

\subsection{Fungal Strains}

T. brevicompactum IBT 40841 (=IBT40841) (IBT Culture Collection, Department of Biotechnology, Technical University of Denmarck, Kongens Lyngby, Denmark) wild type strain and three tri5-overexpressing transformants Tb38tri5, Tb40tri5 and Tb41tri5 [13] were used throughout this study. These strains were maintained on potato dextrose agar medium (PDA) (Difco Becton Dickinson, Sparks, MD, USA).

\subsection{Media and Culture Conditions}

T. brevicompactum mycelia were obtained following a two-step liquid culture procedure [23]. First, the fungus was grown in potato dextrose broth medium (PDB) (Difco Becton Dickinson, Sparks, MD, USA) at $28{ }^{\circ} \mathrm{C}$ for $36 \mathrm{~h}$ and $150 \mathrm{rpm}$. The fungal biomass was harvested by filtration through $30 \mu \mathrm{m}$ pore diameter nylon filters (Sefar AG, Heiden, Switzerland), and the mycelium transferred to minimal medium (MM) [24], containing $20 \mathrm{~g} / \mathrm{L}$ glucose and/or supplemented with 0.25 or $5 \mathrm{mM}$ of the antioxidant tyrosol when indicated. Cultures were maintained at $28{ }^{\circ} \mathrm{C}$ on a rotary shaker at $150 \mathrm{rpm}$ for 1 or 3 days. Biomass was recovered by filtration, washed twice with sterile saline solution $(9 \mathrm{~g} / \mathrm{L}$ $\mathrm{NaCl}$ ), frozen in liquid nitrogen, and stored at $-80{ }^{\circ} \mathrm{C}$ until RNA extraction.

\subsection{DNA and RNA Manipulations}

Standard molecular techniques were performed throughout [25]. RNA extraction was carried out using the TRIZOL reagent (Invitrogen Life Technologies, Carlsbad, CA, USA) according to the manufacturer's instructions from $30 \mathrm{mg}$ mycelium. The integrity and pureness of the RNA was determined using the Agilent 2100 Bioanalyzer (Agilent Technologies, Santa Clara, CA, USA) and cDNA was synthesized with Superscript Choice System for cDNA (Invitrogen Life Technologies, Carslbad, CA, USA) using the Reverse Transcription System (Promega, Madison, WI, USA) and then quantified by determining the absorbance at $260 \mathrm{~nm}$ on a spectrophotometer. 


\subsection{Gene Expression Studies}

Quantitative real-time PCR was performed using the Step One Plus ${ }^{\mathrm{TM}}$ system (Applied Biosystems, Foster City, CA, USA) with Brilliant SYBR Green QPCR Master Mix (Stratagene, La Jolla, CA, USA). All PCR reactions were performed in triplicate in a total volume of $12 \mu \mathrm{L}$ for 40 cycles under the following conditions: denaturation, $95{ }^{\circ} \mathrm{C}, 15 \mathrm{~s}$; annealing, $60{ }^{\circ} \mathrm{C}, 1 \mathrm{~min}$; extension, $72{ }^{\circ} \mathrm{C}, 1 \mathrm{~min}$; and final extension, $72{ }^{\circ} \mathrm{C}, 3 \mathrm{~min}$. Two different sets of cDNA were used for each sample. Threshold cycles (CT) were determined using the 7000 SDS System Software, and CT values were calculated using the $\beta$-tubulin gene as housekeeping control. Data are expressed as $2^{-\Delta \Delta C T}$ [26]. Six values per sample were used for statistical analysis. The primers used in this work are shown in Table 1.

Table 1. Oligonucleotides used in the Real-Time PCR experiments.

\begin{tabular}{|l|l|l|}
\hline Gene & Name & Sequence $\left(\mathbf{5}^{\prime}\right.$-3 $^{\prime}$ ) \\
\hline erg1 & erg1-f & CGCTCCGTGCTTCTTCTC \\
\hline erg1 & erg1-r & CTTCTTCTCTCCCGTCTCC \\
\hline tri3 & Tri3-F & CATTCAGCCACCTAACCTAACCG \\
\hline tri3 & Tri3-R & CCATCCTTCAACCACCGTCGGC \\
\hline tri4 & Tri4-F & CTTGATGGAGCCTTCTCAGC \\
\hline tri6 & Tri4-R & CATCAAGATAGTCCTTATGTTC \\
\hline tri6 & Tri6-F & CGTGCTGACGTGGTTCGAGTGC \\
\hline tri10 & Tri6-R & CTATGGAATGGGTCGGCGAATC \\
\hline tri10 & Tri10-F & CGCTCTCATATGAGTACGTTGGC \\
\hline tri11 & Tri11-F & CGCGTGAATGGTGAAGATGGGC \\
\hline tri11 & Tri11-R & GCAGAGCGCACTTCTTCAGTC \\
\hline tri12 & Tri12-F & GTTCCATATCTTCCGCCATATTC \\
\hline tri12 & Tri12-R & GCGATTGACAGAAGCCATTGC \\
\hline tri14 & Tri14-F & GCTGATGCTGAGCTTGCAAGTG \\
\hline tri14 & Tri14-R & GCCAAGAGGCTCTTGGACGAAG \\
\hline$\beta$-Tubulin & T-tub-F & GAATATCAACAATACCAGGATGG \\
\hline$\beta$-Tubulin & T-tub-R & AGGATTGGTATTGATCATCAGCA \\
\hline
\end{tabular}

\subsection{Tomato Seedling Assay}

The effect of Trichoderma strains (wild-type and tri5-overexpressing transformants) on tomato seedlings was evaluated in vitro. Fungal spore densities of $1 \times 10^{6}$ spores were inoculated by placing the spores at the opposite end of Murashige and Skoog (MS) medium plates (Duchefa Biochemie B.V., Haarlem, The Netherlands), supplemented with $1 \%$ (w/v) sucrose and $8 \mathrm{~g} / \mathrm{L}$ agar, $\mathrm{pH} 5.7$, containing 3-day-old germinated tomato seedlings (5 seedlings per plate). Plates were sealed with flexible film (Parafilm) (Pechiney, Menasha, WI, USA), placed vertically to allow root growth along the agar surface and to allow unimpeded aerial growth of the hypocotyls, and cultured in a growth chamber under conditions of $40 \%$ humidity, $24{ }^{\circ} \mathrm{C}$, and a $16 \mathrm{~h}$ light $/ 8 \mathrm{~h}$ dark photoperiod at $80-100 \mu \mathrm{E} \mathrm{m}^{-2} \mathrm{~s}^{-1}$. MS plates, containing only tomato seedlings, without Trichoderma spores were used as control. 
Experiments were performed in triplicate and measurements of plant size, main root length and number of lateral roots were taken 10-days after Trichoderma inoculation.

\section{Results}

\subsection{Effect of tri5 Gene Overexpression on the Expression of Trichodermin Biosynthetic Genes}

The expression of seven genes (tri4, tri3, tril1, tri6, tri10, tril2 and tri14) of the trichodermin biosynthetic pathway was analyzed from RNAs extracted from mycelia of the wild-type strain IBT40841 and three selected transformants overexpressing the tri5 gene: Tb38tri5, Tb40tri5 and Tb41tri5, incubated 3 days in MM [24] supplemented with $20 \mathrm{~g} / \mathrm{L}$ glucose as described above.

The highest levels of expression were observed in the Tb41tri5 transformant, a strain that produces 2.8-folds more trichodermin than the wild type strain [13]. Expression of tril4 was slightly lower in this transformant that in the wild-type strain (Figure 1).

Figure 1. RT-PCR quantification of expression of seven genes from the trichothecene biosynthetic pathway (tri genes) in the wild-type strain IBT40841 and three tri5-overexpressing transformants Tb38tri5, tb40tri5 and Tb41tri5. The basal condition used for relative measurements was MM without glucose $\left(2^{-\Delta \Delta \mathrm{Ct}}=1\right)$. IBT40841 $\beta$-tubulin was used as an internal reference gene.

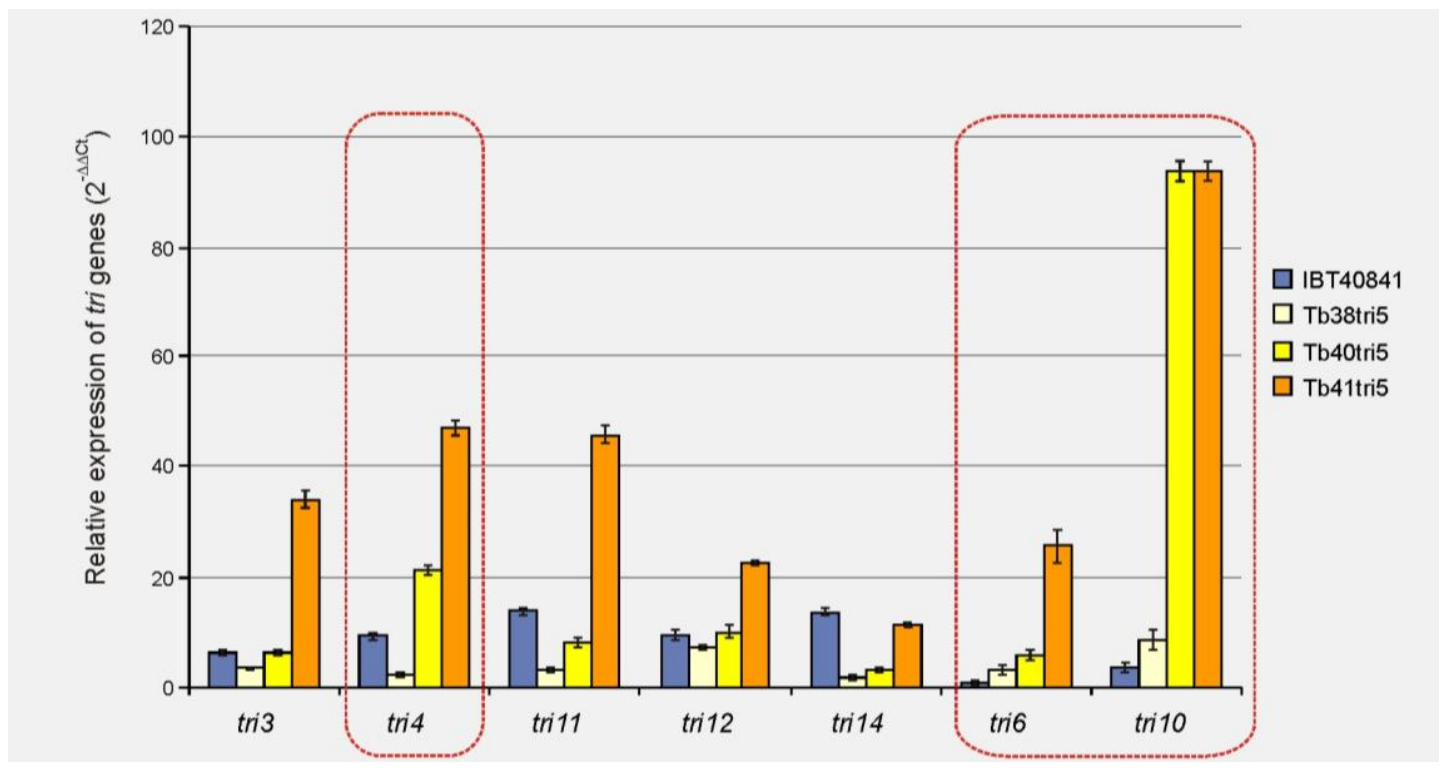

The expression values of the tri3, tri4, trill and tri12 genes in the Tb38tri5 transformant were similar to or lower than those of the wild-type strain. No large differences were observed in the expression levels of the tri3 and tri12 genes between the wild-type and the Tb40tri5 strains. However, the trill and tril4 genes showed a lower level of expression, and the tri4 gene showed a considerably higher level of expression in Tb40tri5 than in the wild-type strain. In the case of the regulatory genes, tri6 and tri10, the level of expression was higher in all the transformants than in the wild-type strain, the greatest differences being seen in the values of tril0 gene expression between the wild-type and the Tb40tri5 and Tb41tri5 transformants. It was previously observed under identical growth conditions that the transformants Tb38tri5, Tb40tri5 and Tb41tri5 showed higher tri5 transcript levels than the 
wild-type strain [13], and although the transformation cassette had been inserted twice into the genomes of the three tri5-overexpressing transformants, they were inserted in different genome locations in the different transformants.

\subsection{Effect of Tyrosol on the Expression of the tri4, tri6 and tri10 Genes in the tri5 Overexpressing}

\section{Transformants}

The different strains were grown for 1 or 3 days in MM [24], with $20 \mathrm{~g} / \mathrm{L}$ glucose, supplemented with 0.25 or $5 \mathrm{mM}$ of the antioxidant tyrosol. The pattern of expression of the tri4, tri6 and trilo genes was similar for the two tyrosol concentrations (Figure 2).

Figure 2. RT-PCR quantification of (A) tri4, (B) tri6 and (C) tril0 gene expression in the wild-type strain and three tri5 overexpressing transformants. The experiment was carried out with mycelia grown for 1 or 3 days in MM [24], with 0.25 or $5 \mathrm{mM}$ tyrosol. The basal condition and the internal reference genes used were those described in Figure 1.

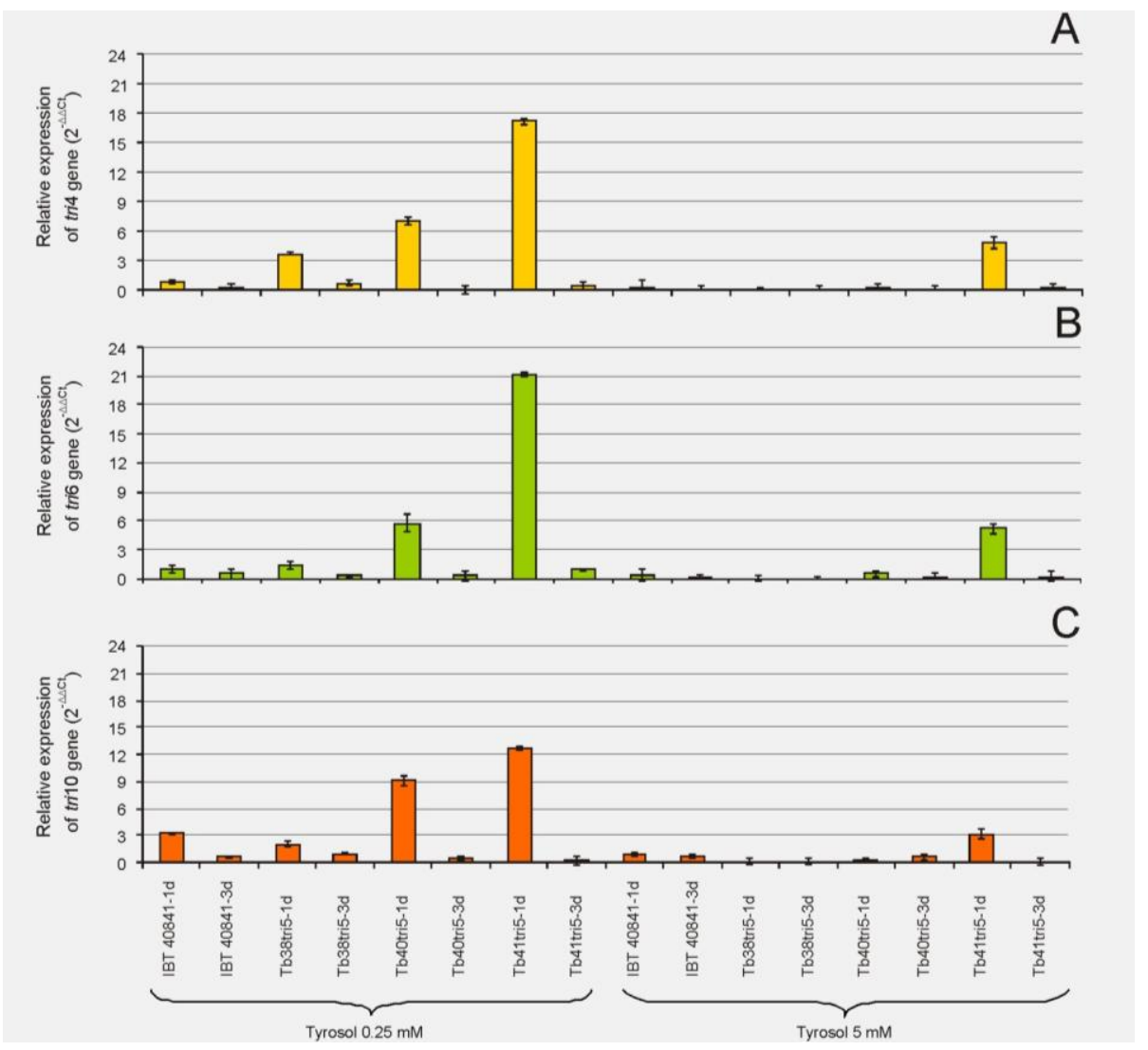

In general, all the genes were repressed by the addition of tyrosol in comparison with the use of MM lacking tyrosol. All the genes showed a higher level of expression after 1 day of growth. In addition, the expression of the genes was higher when the lower concentration of tyrosol was used. The greatest differences in the expression levels were observed between the wild-type strain and the transformants Tb40tri5 and Tb41tri5 after 1 day of growth in the presence of $0.25 \mathrm{mM}$ tyrosol. 


\subsection{Effect of the Overexpression of the Tbtri5 Gene in the Expression of erg1 Gene}

The ergl gene encodes the enzyme squalene epoxidase, which is involved in the biosynthesis of triterpenes [22]. These compounds are synthesized through a pathway common to the trichothecenes up to FPP, from which the pathway diverges to give rise to diterpenes, sesquiterpenes (i.e., trichothecenes) and triterpenes (i.e., ergosterol). The expression levels of ergl were analyzed by real-time PCR from mycelia grown over 3 days in MM supplemented with $20 \mathrm{~g} / \mathrm{L}$ glucose. Higher levels of expression of the erg1 gene were found in the Tb40tri5 and Tb41tri5 transformants in comparison with the wild-type strain (Figure 3A).

Figure 3. RT-PCR quantification of the ergl transcript, in the wild-type strain IBT40841 and three tri5 overexpressing transformants. The strains were grown (A) for 3 days in MM [24], supplemented with $20 \mathrm{~g} / \mathrm{L}$ glucose or (B) for 1 or 3 days in MM, supplemented with $20 \mathrm{~g} / \mathrm{L}$ glucose and $0.25 \mathrm{mM}$ tyrosol. The basal condition and the internal reference genes used were those described in Figure 1.
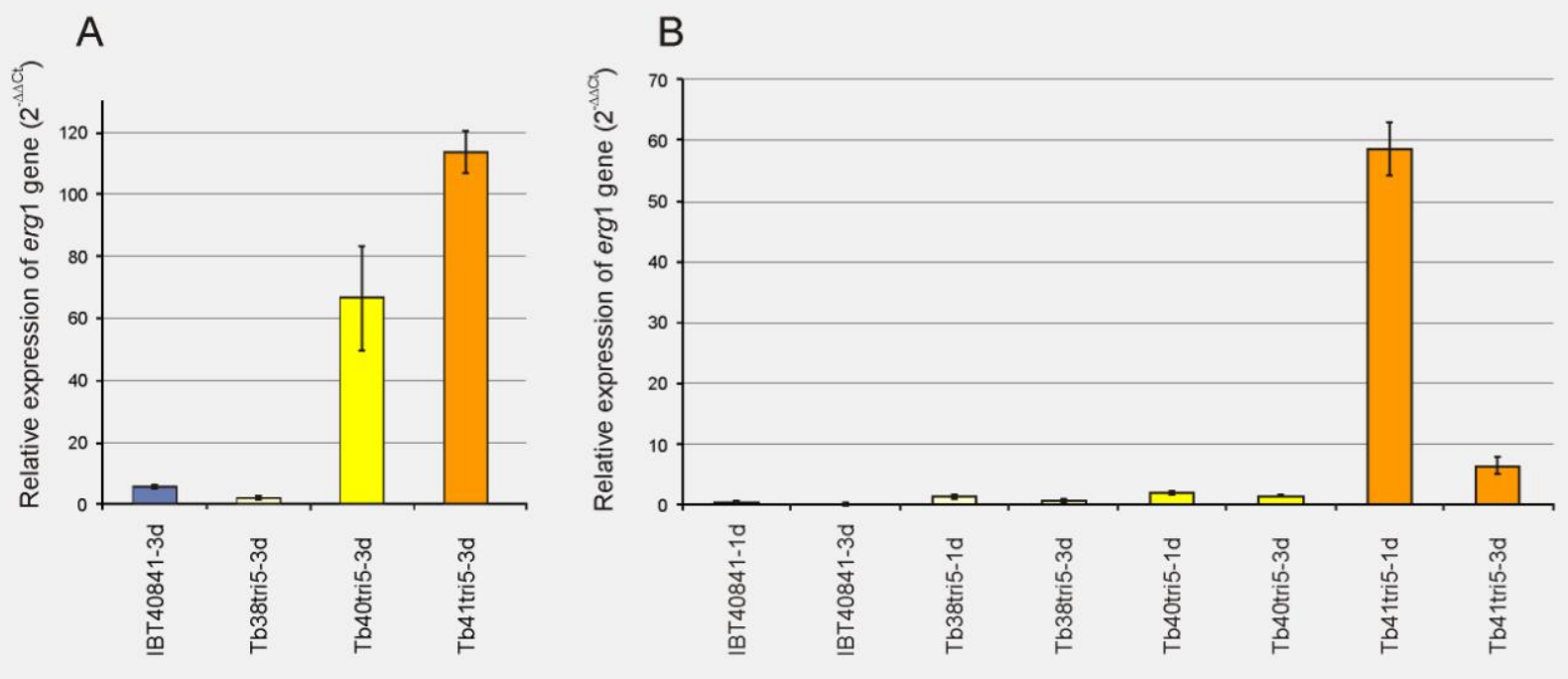

Finally, the effect of the antioxidant agent tyrosol on the expression of ergl gene was analyzed in the wild-type and in the tri5 overexpressing strains. Thus, the different strains were grown for 1 or 3 days in MM, supplemented with $20 \mathrm{~g} / \mathrm{L}$ glucose and $0.25 \mathrm{mM}$ tyrosol. The expression levels showed that in all the strains assayed, expression of the ergl gene was reduced in the presence of tyrosol. The greatest differences in ergl expression levels were found in the Tb40tri5 and Tb41tri5 transformants grown in the presence of $0.25 \mathrm{mM}$ tyrosol in comparison with those transformants grown without tyrosol its absence (Figure 3B).

\subsection{Effect of the tri5 Overexpression on Tomato Seedlings}

An in vitro assay was performed to determine the effect of tri5 overexpression on the development of tomato seedlings, using the wild-type strain and the three tri5-overexpressing transformants.

Ten days after the inoculation of plates containing 3-day-old germinated tomato seedlings with the different Trichoderma strains or with water (control), three parameters were measured for each condition: plant size, length of the main root and number of lateral roots. 
The mean value of plant sizes was $4.1 \pm 1 \mathrm{~cm}$ when they were grown in the presence of water (control); $4 \pm 0.6 \mathrm{~cm}$ in the presence of the wild type strain; $3.7 \pm 0.5 \mathrm{~cm}$ with Tb38tri5; $3.8 \pm 1 \mathrm{~cm}$ with Tb40tri5 and $3.6 \pm 0.4 \mathrm{~cm}$ with Tb41tri5. Statistically significant differences were observed between the size of the control plants and those growing in the presence of the three transformants $(p=0.05)$, and also, between the size of the plants growing in the presence of the wild-type strain and those grown in the presence of Tb38tri5 $(p=0.046)$ and Tb41tri5 $(p=0.045)$. The data indicated that both the presence of $T$. brevicompactum in the growth medium and the tri5 gene overexpression negatively affected to the size of the tomato seedlings.

Root length was also lower when the tomato seedlings were grown in the presence of IBT40841 or the tri5-overexpressing transformants in comparison with the control condition without Trichoderma in the medium. The mean values were: $6.6 \pm 2.0 \mathrm{~cm}$ in the control; $5.1 \pm 1.0 \mathrm{~cm}$ with the $T b 41$ strain; $4.8 \pm 0.8 \mathrm{~cm}$ with Tb38tri5; $5.9 \pm 1.2 \mathrm{~cm}$ with Tb40tri5; and $5.6 \pm 1.0 \mathrm{~cm}$ with Tb41tri5. Finally, it was observed that the number of lateral roots, in comparison with the control, was similar or was reduced when wild-type IBT40841 or the tri5-overexpressing transformants were present in the medium used to grow the tomato seedlings. The mean values were: $15.9 \pm 8.0$ in the control, $11.5 \pm 5.2$ with the wild type strain, $11.1 \pm 4.2$ with Tb38tri5, $15.9 \pm 4.6$ with Tb40tri5 and $14.8 \pm 3.0$ with Tb41tri5. The greatest reduction in the number of lateral roots with respect to the control was seen in the presence of the wild-type strain or the Tb38tri5 transformant (Figure 4). In both determinations (root length and number of lateral roots), statistically significant differences were seen between the control condition and the conditions that contained Trichoderma $(p \leq 0.05)$, and also between the condition treated with the wild-type strain and those treated with spores from the three tri5-overexpressing transformants $(p=0.05)$ (Figure 4).

Figure 4. Effect of T. brevicompactum and of tri5 overexpressed transformants on tomato seedlings. Three-day-old germinated tomato seedlings grown on MS were inoculated with (A) water; (B) IBT40841; (C) Tb38tri5; (D) Tb40tri5; or (E) Tb41tri5. Photographs were taken 10-days after inoculating $1 \times 10^{6}$ Trichoderma spores.
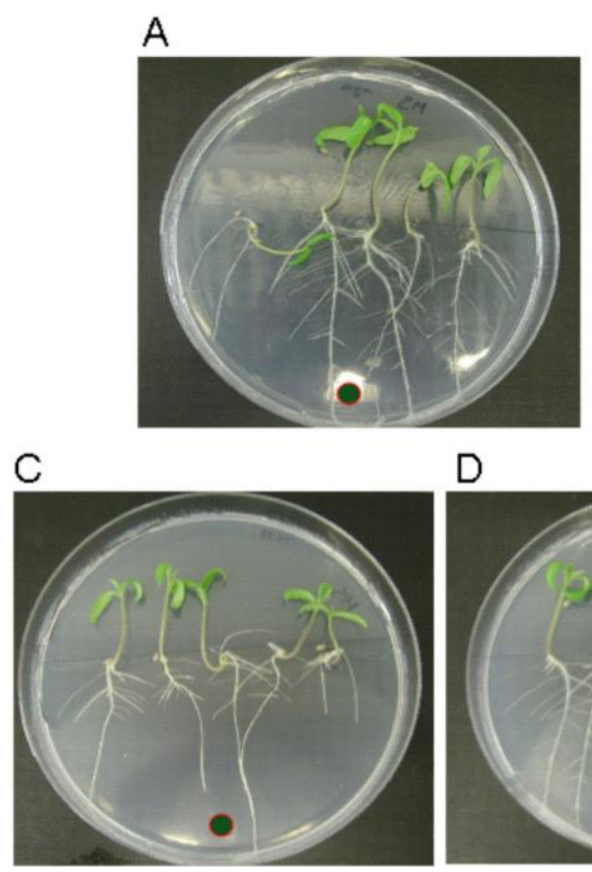

D
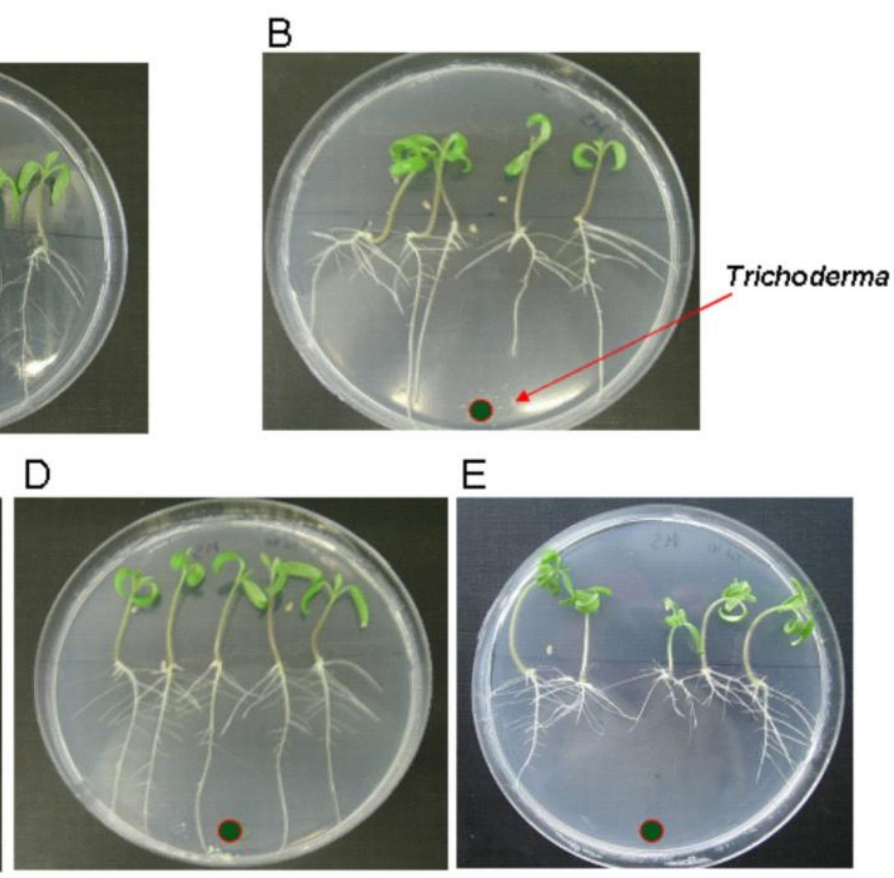

$\mathrm{E}$

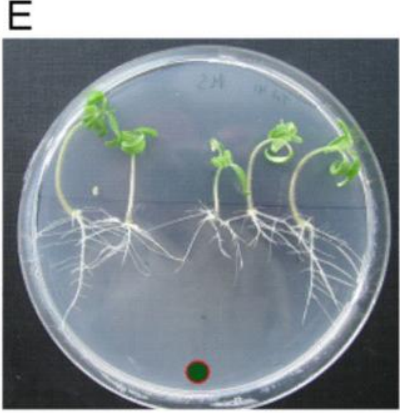




\section{Discussion}

Overexpression of the IBT40841 tri5 gene resulted in an increase of the trichodermin production as a result of a higher level of tri5 transcript and also in an increase in the antibiotic activity against a large panel of yeast, and it negatively affected tomato plant growth and the lesions caused by Botrytis cinerea [13].

Two of the tri5 gene overexpression transformants used in the present work, Tb40tri5 and Tb41tri5, had an increased level of expression of the regulatory genes tri6 and tri10, and tri4, the oxygenase gene that controls conversion of trichodiene to trichodermol [11]. However, in the Tb41tri5 transformant, the expression of tri3, trill and tril2 genes were also induced. In spite of these differences between the different transformants it is likely that the overproduction of trichodiene has a positive effect on the expression of the tri4 gene, the next gene in the biosynthetic pathway (Figure 5), but also in the expression of the tri6 and tri 10 genes, that would regulate the expression of all the tri genes.

Figure 5. Schematic representation of the model of regulation of the trichothecenes harzianum A and trichodermin biosynthesis. The synthesis of diterpenes and triterpenes is indicated by yellow squares. The genes indicated in red are those studied in the present work. tri6 and tri10 are regulatoy genes. Farnesol and tyrosol are two regulatory and antagonistic molecules. The red arrows indicated positive regulation. The blue doted lines indicate possible inhibitory activities (Modified from [12,27-30]).

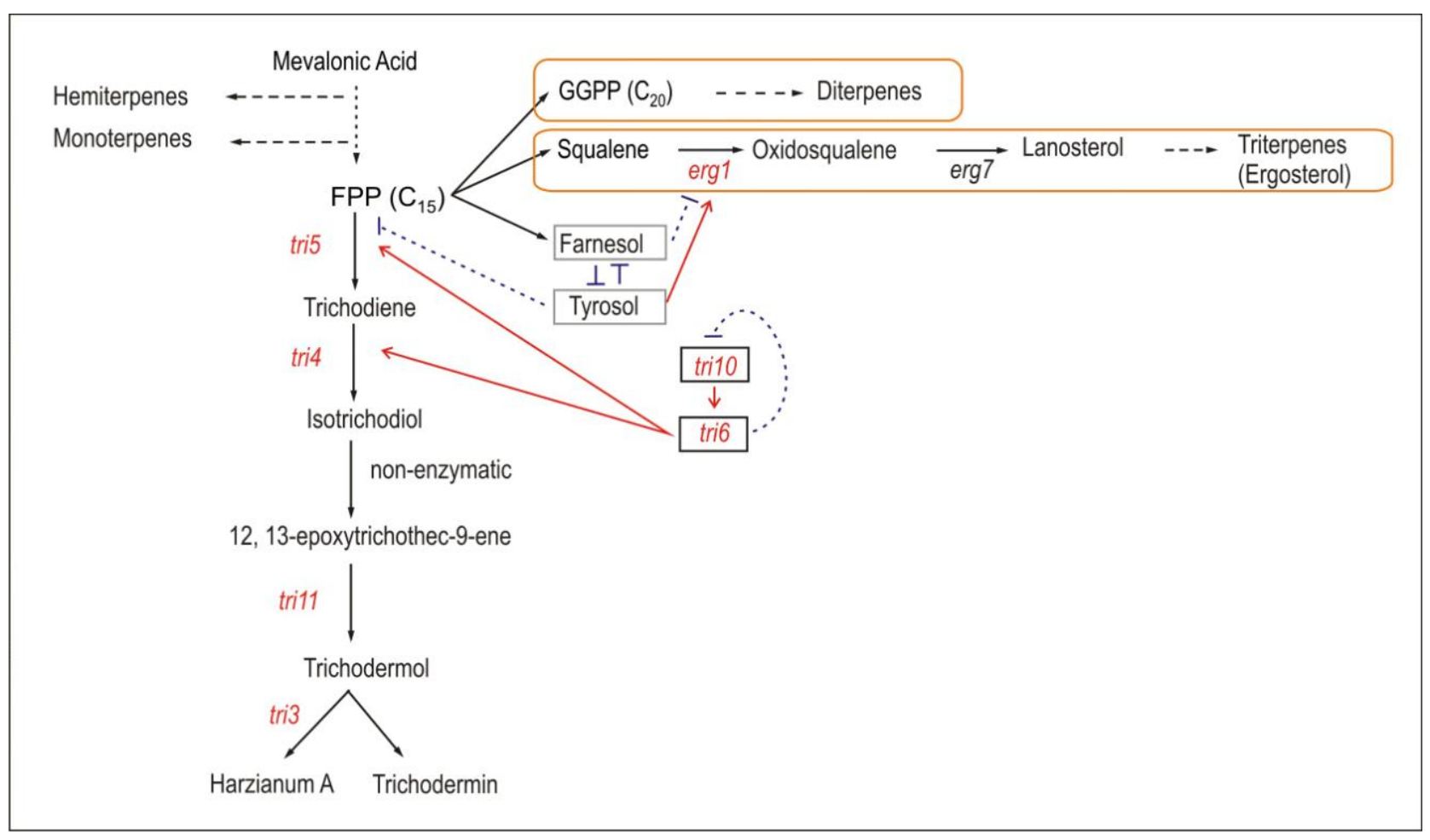

The production of tyrosol and hydroxytyrosol simultaneous to the increase of the trichodermin level in the tri5-overexpressing transformants can be explained as a response by cells to regulate the levels of farnesol:tyrosol, since both trichodiene and farnesol have FPP as precursor. When farnesol and tyrosol are present in direct competition, tyrosol does not alter the quorum-sensing activity of farnesol, even when the tyrosol is present at a 16-fold molar excess [31]. When tyrosol was added to the culture 
media, the expression of the tri5, tri4, tri6 and tril0 genes was repressed, and this repressing effect persisted until at least the seventh day of culture (data not shown). However, this repressing effect of tyrosol on tri gene expression was observed only when this compound was added to the culture medium but when tyrosol was produced by the tri5-overexpressing transformants, rather, there was a 2.4 fold increase in toxin production in the transformant Tb41tri5 when compared to the wild-type strain [13]. Thus, without external addition of tyrosol a significant increase in the expression of the tri genes was observed in the transformants (see Figure 1), probably because the effect of the endogenous tyrosol would be regulated by the intracellular farnesol, equilibrating both stimulating and repressing activities, respectively. The addition of external tyrosol would shift this equilibrium towards the stimulating effects of the tyrosol, resulting in a repression of the genes involved in the biosynthesis of trichodermin, as a secondary metabolite, and a stimulation of the primary metabolism biosynthetic pathways. In this sense, the increase in the level of expression of the ergl gene in the tri5 overexpressing transformants can be understood in the same way, since ergl encodes squalene epoxidase, the enzyme that uses squalene as substrate in the first step of the ergosterol biosynthesis. Squalene is the condensation product of two farnesyl pyrophophates. Thus, the increase in the level of expression of ergl gene will direct the FPP towards the ergosterol biosynthesis, which is needed for the fungal cell growth, one of the stimulating effects exerted by the tyrosol. This correlates well with the previous observation that farnesol biosynthesis inhibits the ergosterol pathway in C. albicans [32].

Many Trichoderma species maintain a relationship with the plants as avirulent symbionts [33], with benefits that include better root development, increase in crop production and defense stimulation [34]. Secondary metabolites produced by Trichoderma have stimulant effects on plant development when they are applied at low doses [35]. However, it has been described that Fusarium spp. trichothecenes are phytotoxic and that they act as virulence factors in host-compatible plants [36]. Furthermore, it is known that trichothecenes such as harzianum $\mathrm{A}$ and trichodermin, characteristic from the clade Brevicompactum, are strong inhibitors of the seed germination and that they do not promote plant growth owing to their phytotoxicity $[12,37,38]$. The in vitro assays carried out in the present work with tomato seedlings showed that the plant size and the root length were lower in the presence of T. brevicompactum and even lower with the Tb40tri5 and Tb41tri5 transformants. In addition, it has recently been shown in greenhouse assays that tomato seed treatments with $T$. brevicompactum strains increased the leaf necrotic lesions produced by B. cinerea after leaf inoculations with this pathogen, and mainly when the tomato seeds were coated with the tri5-overexpressing transformants, invalidating T. brevicompactum IBT 40841 as a biocontrol agent in agriculture [13].

\section{Conclusions}

In this work we have shown that the overexpression of the tri5 gene resulted in an increase of the level of transcription of three trichothecene genes, tri4, tri6 and tril0 genes, and that tri5 gene may have an important role in the trichothecene-triterpene-diterpene regulatory network, since tri5 expression may contribute to the balance of FPP-farnesol-squalene-trichodiene in the different cell growth stages. We found that growth in the presence of added tyrosol reduced the tri gene expression and increased ergl gene expression, directing the pool of FPP towards biosynthesis of ergosterol, a primary metabolite, instead of towards the synthesis of secondary metabolites like trichothecenes, thus 
avoiding the repressing effect exerted by farnesol. Finally, transformants Tb40tri5 and Tb41tri5 reduced plant growth and main root size to a greater extent than the wild type strain, probably due to the intrinsic phytotoxic effect of the trichodermin, whose production was significantly increased in these transformants.

\section{Acknowledgments}

Research project funding was from Junta de Castilla y León (GR67) and the Spanish Ministry of Science and Innovation (AGL2008-0512/AGR, AGL2009-13431-C01 and AGL2009-13431-C02). AT was granted by a Spanish Foreing Office AECID award. MGM was granted with a FPU fellowship by the Spanish Ministry of Science and Innovation (AP2007-02835). Thanks to Ulf Thrane for providing us the strain T. brevicompactum IBT 40841 and Elias R. Olivera for critical reading of the article.

\section{References}

1. Monte, E. Understanding Trichoderma: Between biotechnology and microbial ecology. Int. Microbiol. 2001, 4, 1-4.

2. Weindling, R. Trichoderma lignorum as a parasite of other soil fungi. Phytopathology 1932, 22, 837-845.

3. Kubicek, C.P.; Penttilä, M. Regulation of Production of Plant Polysacchride Degrading Enzymes by Trichoderma. In Trichoderma and Gliocladium: Enzymes, Biological Control and Commercial Applications; Kubicek, C.P., Harman, G.E., Eds.; Taylor \& Francis: London, UK, 1998; Volume 2, pp. 49-71.

4. Hjeljord, L.; Tronsmo, A. Trichoderma and Gliocladium in Biological Control: An Overview. In Trichoderma and Gliocladium: Enzymes, Biological Control and Commercial Applications; Kubicek, C.P., Harman, G.E., Eds.; Taylor and Francis: London, UK, 1998; Volume 2, pp. 131-153.

5. Vizcaíno, J.A.; Sanz, L.; Basilio, A.; Vicente, F.; Gutiérrez, S.; Hermosa, M.R.; Monte, E. Screening of antimicrobial activities in Trichoderma isolates representing three Trichoderma sections. Mycol. Res. 2005, 109, 1397-1406.

6. Goldstein, J.L.; Brown, M.S. Regulation of the mevalonate pathway. Nature 1990, 343, 425-430.

7. Chang, M.C.; Keasling, J.D. Production of isoprenoid pharmaceuthicals by engineering isoprenoids. Nat. Chem. Biol. 2006, 2, 674-681.

8. Kirby, J.; Keasling, J.D. Biosynthesis of plant isoprenoids: Perspectives for microbial engineering. Annu. Rev. Plant Biol. 2009, 60, 335-355.

9. Beremand, M.N.; McCormick, S.P. Biosynthesis and Regulation of Trichothecene Production by Fusarium Species. In Handbook of Applied Mycology. Mycotoxins in Ecological Systems; Bhatnagar, D., Lilehoj, E.B., Arora, D.K., Eds.; Marcel Dekker, Inc.: New York, NY, USA, 1992; Volume 5, pp. 360-389.

10. Kimura, M.; Tokai, T.; Takahashi-Ando, N.; Ohsato, S.; Fujita, M. Molecular and genetic studies of Fusarium trichothecene biosynthesis: Pathways, genes, and evolution. Biosci. Biotechnol. Biochem. 2007, 71, 2105-2123. 
11. Cardoza, R.E.; Malmierca, M.G.; Hermosa, M.R.; Alexander, N.J.; McCormick, S.P.; Proctor, R.H.; Tijerino, A.M.; Rumbero, A.; Monte, E.; Gutiérrez, S. Identification of loci and functional characterization of trichothecene biosynthetic genes in the filamentous fungus Trichoderma. Appl. Environ. Microbiol. 2011, 77, 4867-4877.

12. Degenkolb, T.; Dieckmann, R.; Nielsen, K.F.; Gräfenhan, T.; Theis. Ch.; Zafari, D.; Chaverri, P.; Ismaiel, A.; Brückner, H.; Von Döhren, H.; et al. The Trichoderma brevicompactum clade: A separate lineage with new species, new peptaibiotics, and mycotoxins. Mycol. Prog. 2008, 7, 177-219.

13. Tijerino, A.; Cardoza, R.E.; Moraga, J.; Malmierca, M.G.; Vicente, F.; Aleu, J.; Collado, I.G.; Gutiérrez, S.; Monte, E.; Hermosa, R. Overexpression of the trichodiene synthase gene tri5 increases trichodermin production and antimicrobial activity in Trichoderma brevicompactum. Fungal Genet. Biol. 2011, 48, 285-296.

14. Brown, D.W.; Dyer, R.B.; McCormick, S.P.; Kendra, D.F.; Plattner, R.D. Functional demarcation of the Fusarium core trichothecene gene cluster. Fungal Genet. Biol. 2004, 41, 454-462.

15. Trapp, S.C.; Hohn, T.M.; McCormick, S.; Jarvis, B.B. Characterization of the gene cluster for biosynthesis of macrocyclic trichothecenes in Myrothecium roridum. Mol. Gen. Genet. 1998, 257 , 421-432.

16. Tarus, P.K.; Langát-Thoruwa, C.C.; Wanyonyi, A.W.; Chhlabra, S.C. Bioactive metabolites from Trichoderma harzianum and Trichoderma longibrachiatum. Bull. Chem. Soc. Ethiop. 2003, 17, 185-190.

17. Alem, M.A.; Oteef, M.D.; Flowers, T.H.; Douglas, L.J. Production of tyrosol by Candidda albicans biofilms and its role in quorum sensing and biofilm development. Eukaryot. Cell 2006, 5, 1770-1779.

18. Daoubi, M.; Pinedo-Rivilla, C.; Rubio, M.B.; Hermosa, R.; Monte, E.; Aleu, J.; Collado, I.G. Hemisynthesis and absolute configuration of novel 6-pentyl-2H-pyran-2-one derivatives from Trichoderma spp. Tetrahedron 2009, 65, 4834-4840.

19. Sentheshanmuganathan, E.; Elsden, S.R. The mechanism of the formation of tyrosol by Saccharomyces cerevisiae. Biochem. J. 1958, 69, 210-218.

20. Covas, M.I.; Miró-Casas, E.; Fitó, M.; Farré-Albadalejo, M.; Gimeno, E.; Marrugat, J.; de la Torre, R. Bioavailability of tyrosol, an antioxidant phenolic compound present in wine and olive oil, in humans. Drug. Exp. Clin. Res. 2003, 29, 203-206.

21. Ahn, E.Y.; Jiang, Y.; Zhang, Y.; Son, E.M.; You, S.; Kang, S.W.; Pardk, J.; Jung, J.H.; Lee, B.J.; Kim, D. Citotoxicity of $p$-tyrosol and its derivatives may correlate with the inhibition of DNA replication initiation. Oncol. Rep. 2008, 19, 527-534.

22. Cardoza, R.E.; Vizcaíno, J.A.; Hermosa, M.R.; Sousa, S.; González, F.J.; Llobell, A.; Monte, E.; Gutiérrez, S. Cloning and characterization of the ergl gene of Trichoderma harzianum: Effect of the ergl silencing on ergosterol biosynthesis and resistance to terbinafine. Fungal Genet. Biol. 2006, 43, 164-178.

23. Vizcaíno, J.A.; Cardoza, R.E.; Dubost, L.; Bodo, B.; Gutiérrez, S.; Monte, E. Detection of peptaibols and partial cloning of a putative peptaibol synthetase gene from T. harzianum CECT 2413. Folia Microbiol. 2006, 51, 114-120.

24. Penttilä, M.; Nevalainen, H.; Ratto, M.; Salminen, E.; Knowles, J. A versatile transformation system for the cellulolytic filamentous fungus Trichoderma reesei. Gene 1987, 61, 155-164. 
25. Sambrook, J.; Russel, D.W. Molecular Cloning; Cold Spring Harbor Laboratory Press: New York, NY, USA, 2001.

26. Livak, K.J.; Schmittgen, T.D. Analysis of relative gene expression data using real-time quantitative PCR and the 2(-Delta Delta CT). Methods 2001, 25, 402-408.

27. Desjardins, A.E.; Hohn, T.M.; McCormick, S.P. Trichothecene biosynthesis in Fusarium species: Chemistry, genetics, and significance. Microbiol. Rev. 1993, 57, 595-604.

28. De Sordi, L.; Mühlschlegel, F.A. Quorum sensing and fungal-bacterial interactions in Candida albicans: A communicative network regulating microbial coexistence and virulence. FEMS Yeast Res. 2009, 9, 990-999.

29. Tokai, T.; Koshino, H.; Takahashi-Ando, N.; Sato, M.; Fujimura, M.; Kimura, M. Fusarium tri4 encodes a key multifunctional cytochrome P450 monooxygenase for four consecutive oxygenation steps in trichothecene biosynthesis. Biochem. Biophs. Res. Commun. 2007, 353, 412-417.

30. Kruppa, M. Quorum sensing and Candida albicans. Mycoses 2009, 51, 1-10.

31. Nickerson, K.W.; Atkin, A.L.; Hornby, J.M. Quorum sensing in Dimorphic fungi: farnesol and beyond. Appl. Environ. Microbiol. 2006, 72, 3805-3813.

32. Hornby, J.M.; Kebaara, B.W.; Nickerson, K.W. Farnesol biosynthesis in Candida albicans: Cellular response to sterol inhibition by zaragozic acid. B. Antimicrob. Agents Chemother. 2003, 47, 2366-2369.

33. Harman, G.E.; Howell, C.R.; Viterbo, A.; Chet, I.; Lorito, M. Trichoderma species opportunistic, avirulent plant symbionts. Nat. Rev. Microbiol. 2004, 84, 377-393.

34. Harman, G.E. Overview of mechanisms and uses of Trichoderma spp. Phytopathology 2006, 96, 190-194.

35. Vinale, F.; Sivasithamparam, K.; Ghisalberti, E.L.; Marra, R.; Woo, S.L.; Lorito, M. Trichoderma-plant-pathogen interactions in soil agro-ecoystems. Soil Biol. Biochem. 2008, 40, 1-10.

36. Proctor, R.H.; Hohn, T.M.; McCormick, S.P. Reduced virulence of Gibberella zeae caused by disruption of a trichothecene toxin biosynthetic gene. Mol. Plant Microbe Interact. 1995, 8, 593-601.

37. Cutler, H.G.; LeFiles, J. Trichodermin: Effects on plants. Plant Cell Physiol. 1978, 19, 177-182.

38. Nielsen, K.F.; Gräfenhan, T.; Zafari, D.; Thrane U. Trichothecene production by Trichoderma brevicompactum. J. Agric. Food Chem. 2005, 53, 8190-8196.

(C) 2011 by the authors; licensee MDPI, Basel, Switzerland. This article is an open access article distributed under the terms and conditions of the Creative Commons Attribution license (http://creativecommons.org/licenses/by/3.0/). 